\title{
Minimal change disease and acute interstitial nephritis following SARS-CoV-2 BNT162b2 vaccination
}

\author{
Pondtip Jongvilaikasem ${ }^{1} \cdot$ Pornpimol Rianthavorn ${ }^{2}[$
}

Received: 13 January 2022 / Revised: 13 January 2022 / Accepted: 14 January 2022 / Published online: 7 February 2022

(c) The Author(s), under exclusive licence to International Pediatric Nephrology Association 2022

To the Editors,

We read with interest the report from Niel et al. [1] describing IgA nephropathy following messenger RNA (mRNA) vaccination for coronavirus disease-2019 (COVID-19). Pfizer-BioNTech SARS-CoV-2 BNT162b2 mRNA vaccine has met efficacy and safety standards, and received emergency authorization for children aged 5 years and above. The vaccine has been described as a potential trigger of glomerular diseases in adults [2]. Here, we describe new-onset minimal change disease (MCD) and acute interstitial nephritis (AIN) related to the SARS-CoV-2 BNT162b2 vaccine.

A healthy 14-year-old boy developed bilateral leg edema 5 days after the first injection of the SARS-CoV-2 BNT162b2 vaccine. Evaluation revealed hypertension, nephrotic syndrome (proteinuria $4+$ and $9 \mathrm{~g} / \mathrm{g}$ creatinine, albumin $2 \mathrm{~g} / \mathrm{dL}$, and cholesterol $257 \mathrm{mg} / \mathrm{dL}$ ), and acute kidney injury (creatinine $2 \mathrm{mg} / \mathrm{dL}$ ) with negative urine sediment examination. The patient denied taking any medication. The nasopharyngeal swab for SARS-CoV-2 reverse transcriptase-polymerase chain reaction was negative. The edema was progressively worsening. Ten days after vaccination, he became anuric with a peak serum creatinine of $9 \mathrm{mg} /$
dL. Three daily doses of pulse methylprednisolone were administered, followed by oral prednisolone, $60 \mathrm{mg}$ daily. He received hemodialysis for 3 weeks (Fig. 1a). Evaluation for secondary glomerular disease, including $\mathrm{C} 3 / \mathrm{C} 4$, ANA, ANCA, hepatitis B surface antigen, and antibodies to hepatitis $C$ virus, was negative. Renal pathology showed eighteen unremarkable glomeruli by light microscopy and negative immunofluorescence staining. Electron microscopy showed diffuse foot process effacement, consistent with MCD. Diffuse tubular injury and interstitial inflammatory cell infiltration were noted (Fig. 1b-d). After the 5-week treatment of corticosteroids, the patient was in partial remission (creatinine $0.53 \mathrm{mg} / \mathrm{dL}$ and proteinuria $0.9 \mathrm{~g} / \mathrm{g}$ creatinine). Total immunoglobulin specific to the receptor-binding domain of the SARS-CoV-2 spike protein using Elecsys $®$ (Roche Diagnostics, Basel, Switzerland) was $5.6 \mathrm{U} / \mathrm{mL}$ (positive $\geq 0.8 \mathrm{U} /$ $\mathrm{mL}$ ), indicating an antibody response to the vaccine.

Although serious adverse effects of COVID-19 vaccines are rare, it is important to continue to monitor its safety. The only other published pediatric case described a patient with steroid-sensitive nephrotic syndrome and stable kidney function following mRNA COVID-19 vaccination [3]. As MCD related to the SARS-CoV-2 BNT162b2 vaccine often

Pornpimol Rianthavorn

pornpimol.r@chula.ac.th

1 Pediatric Division, Hat Yai Medical Education Center, Hat Yai Hospital, Songkhla, Thailand

2 Division of Nephrology, Department of Pediatrics, Faculty of Medicine, Chulalongkorn University, 1873 King Rama 4 Road, Pathumwan, Bangkok 10330, Thailand 

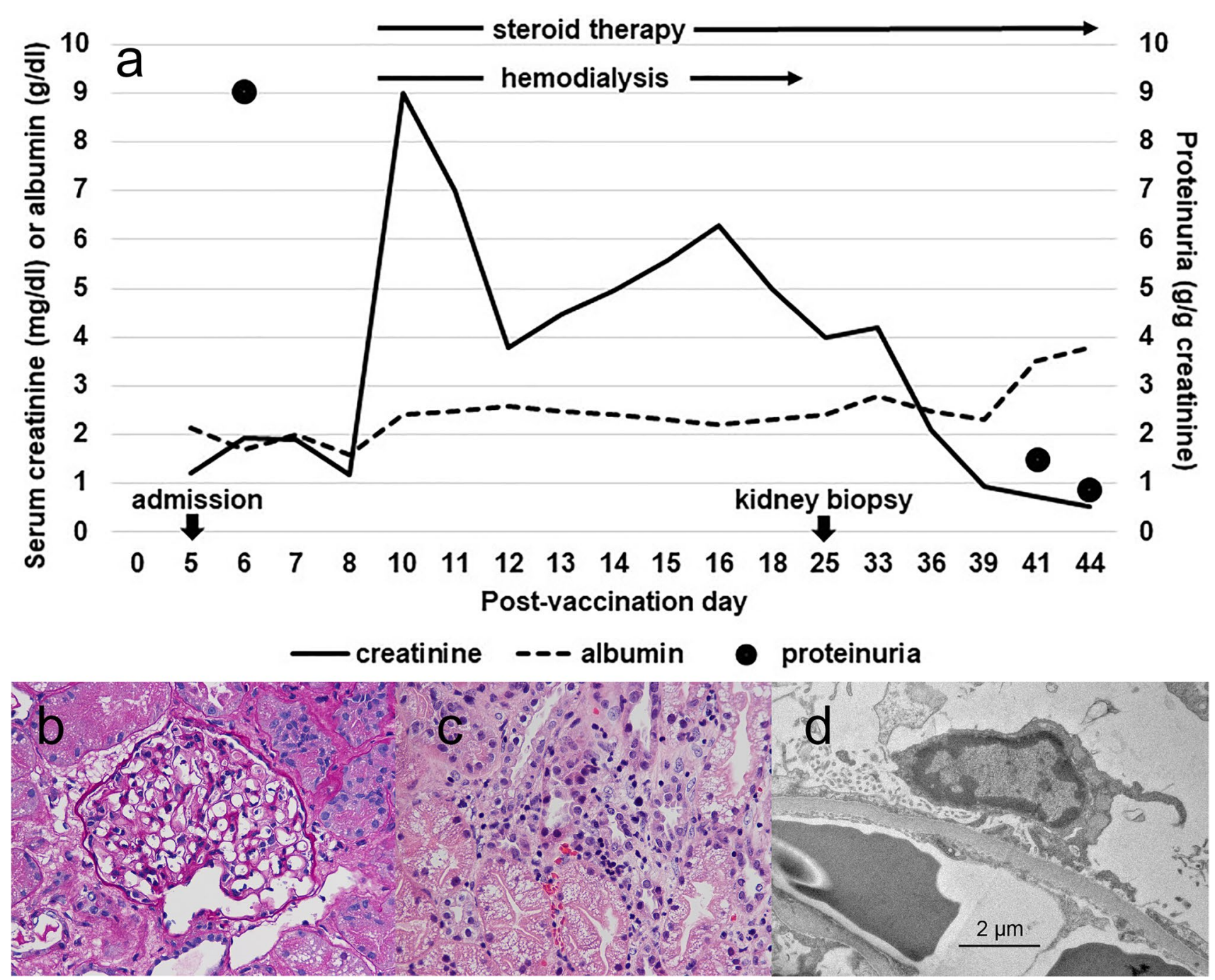

Fig. 1 a The course of serum creatinine, albumin, and proteinuria over 6 weeks after vaccination, b light microscopy shows a histologically unremarkable glomerulus (original magnification $\times 400$ ), c dif-

develops within the first week of vaccination, the strong temporal association suggests that the T-cell mediated immune response to the SARS-CoV-2 BNT162b2 vaccine could be a potential trigger for podocytopathy $[2,3]$. Our case provides additional support for a possible association between the SARS-CoV-2 BNT162b2 vaccine and pediatric kidney disease.

Acknowledgements The authors would like to thank Professor Yong Poovorawan, MD. and Ms. Nuanpan Siripen for their help in the management of the patient.

Author contribution All the authors contributed to drafting, reviewing, and revising this paper. fuse acute tubular injury and interstitial inflammatory cell infiltration (original magnification $\times 400$ ), $\mathbf{d}$ electron microscopy shows podocyte foot process effacement (original magnification $\times 5000$ )

\section{Declarations}

Competing interests The authors declare no competing interests.

Ethics approval This case study involving human participants was in accordance with the ethical standards of the institutional and national research committee and with the 1964 Helsinki Declaration and its later amendments or comparable ethical standards. The Human Investigation Committee (IRB) of Hat Yai Hospital approved this study.

Consent to participate Written informed consent was obtained from the parents.

Consent to publish The participant has consented to the submission of the case report to the journal. 


\section{References}

1. Niel O, Florescu C (2021) IgA nephropathy presenting as rapidly progressive glomerulonephritis following first dose of COVID-19 vaccine. Pediatr Nephrol. https://doi.org/10.1007/ s00467-021-05351-x

2. Wu HHL, Kalra PA, Chinnadurai R (2021) New-onset and relapsed kidney histopathology following COVID-19 vaccination: a systematic review. Vaccines. https://doi.org/10.3390/vaccines91 11252
3. Nakazawa E, Uchimura T, Hirai Y, Togashi H, Oyama Y, Inaba A, Shiga K, Ito S (2021) New-onset pediatric nephrotic syndrome following Pfizer-BioNTech SARS-CoV-2 vaccination: a case report and literature review. CEN Case Rep. https://doi.org/10. 1007/s13730-021-00656-0

Publisher's note Springer Nature remains neutral with regard to jurisdictional claims in published maps and institutional affiliations. 